\title{
Coledocolitiasis y pancreatitis: las dificultades de la predicción clínica
}

\section{Choledocholithiasis and pancreatitis: the difficulties of clinical prediction}

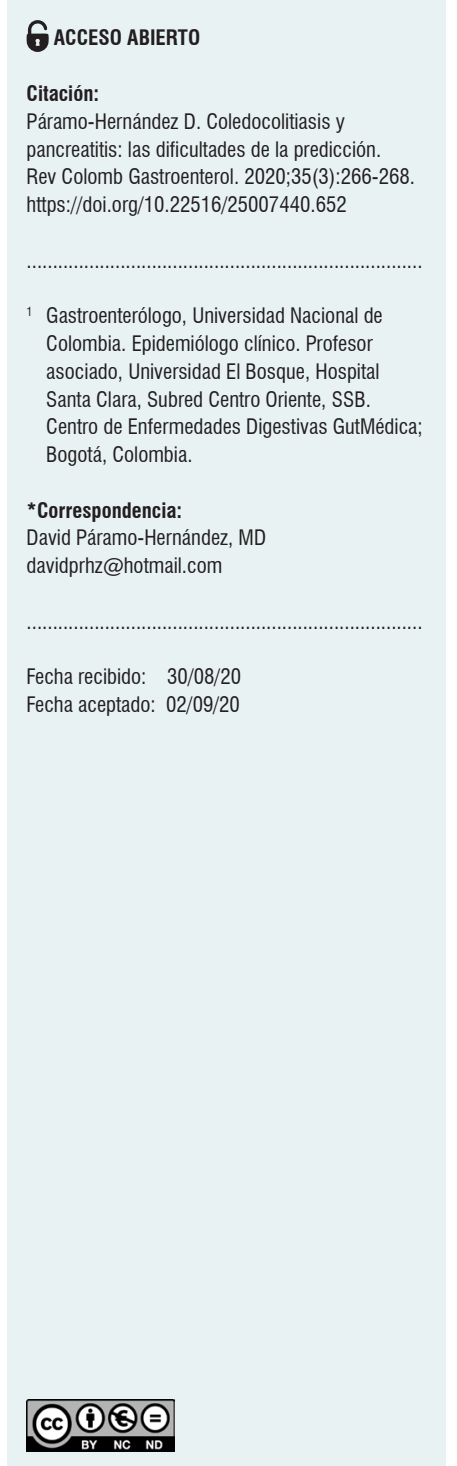

En la práctica clínica, nos hemos enfrentado en muchas ocasiones ante la incertidumbre diagnóstica y las dificultades de elegir (o no) un procedimiento como la colangiopancreatografía retrógrada endoscópica (CPRE), con sus eventuales complicaciones, en el contexto de la coledocolitiasis.

Frente a un panorama impredecible y con un reconocido $50 \%$ de las pancreatitis con origen biliar por coledocolitiasis (1), este número de nuestra revista apunta, precisamente, a explorar la posibilidad de predicción diagnóstica de la coledocolitiasis, tal y como se puede apreciar en los trabajos de Gastelbondo, Toro-Calle, Yurgaky y colaboradores; pero también se apuesta por comparar, valorar y estimar las escalas de riesgo y pronóstico en relación con la morbimortalidad en la pancreatitis, lo cual se desarrolla en el trabajo realizado por Rodríguez-Varón y colaboradores.

La coledocolitiasis es una condición clínica de difícil diagnóstico, que requiere de algoritmos de procedimientos que nos permitan alcanzar una precisión sin mayores complicaciones durante su realización $(2,3)$. La experiencia de validación de estos modelos ha constituido un reto; de hecho, múltiples experiencias muestran resultados que finalmente no son satisfactorios (4). El desafío, entonces, ha consistido en la búsqueda un modelo estadístico predictivo para una adecuada decisión clínica.

Dado que no existe un modelo universal de predicción clínica para una enfermedad, es importante desarrollar modelos específicos separados, que puedan evaluar individualmente el papel de la etnia, la nacionalidad, el sexo o la edad en el riesgo de una patología (5). Se requiere de un tamaño muestral grande, así como de disponer de 2 conjuntos de datos: uno para el desarrollo del modelo y otro para su validación. El desempeño predictivo se logrará a través de múltiples validaciones (5).

Por tanto, es necesario efectuar el manejo de las variables para obtener los predictores, mediante el análisis de regresión múltiple o logística de aquellas que alcancen diferencias significativas. Esto permitirá identificar el conjunto de variables que se constituyan en predictoras del desenlace (3).

Asimismo, se debe evaluar el poder predictivo del modelo propuesto, utilizando un conjunto de datos independientes -preferentemente externos-, siempre que estén disponibles. Existen varias medidas de desempeño, pero los dos componentes clave son la calibración y la discriminación (5).

Los autores, editores y lectores debemos familiarizarnos con algunas herramientas que nos permitan valorar la calidad de los trabajos en los cuales se incluyan el desarrollo, la validación o las actualizaciones de los modelos de predicción clínica. En efecto, 
existe una iniciativa independiente de expertos estadísticos, epidemiólogos y metodólogos -que componen el grupo TRIPOD (Transparent Reporting of a multivariable prediction model for Individual Prognosis Or Diagnosis)-, y emitieron un consenso, así como una lista de chequeo, para la evaluación adecuada de su calidad (6).

El trabajo de Gastelbondo y colaboradores se propuso comparar los criterios de predicción de la coledocolitiasis, desarrollados por la Sociedad Americana de Endoscopia Gastrointestinal (American Society for Gastrointestinal Endoscopy, ASGE) (7), frente a los parámetros de la Sociedad Británica de Gastroenterología (SBG) (8). Esto se realizó a partir de un tamaño muestral obtenido por conveniencia y no probabilístico, dada la intención de comparar estas pruebas diagnósticas.

Así pues, la verificación de la coledocolitiasis se realizó mediante CPRE o colangiografía por resonancia magnética (CRM).La no utilización del ultrasonido endoscópico excluye una alternativa diagnóstica vigente válida, con una exactitud y probabilidades pre y posprueba comparables con la CRM (9); la CPRE fue el método de referencia (gold standard).

Se encontró que, en el grupo de los pacientes con probabilidad alta, la sensibilidad para las guías de la SBG y la ASGE fue del 65 y el $74 \%$, respectivamente, mientras que la especificidad fue del $33 \%$ frente al $28 \%$, y la exactitud alcanzó el 39 y el $67 \%$, respectivamente.

Entre tanto, en el grupo de pacientes con probabilidad intermedia, la sensibilidad para las guías de la SBG y la ASGE fue del 34 y el $35 \%$, respectivamente; la especificidad fue del 66 y el $71 \%$, y la exactitud del 60 y el $32 \%$. En el trabajo de Toro-Calle se observó un rendimiento igual- mente modesto en este mismo grupo de pacientes, a partir de los criterios de la ASGE.

Los hallazgos de los desempeños predictores diagnósticos modestos, encontrados en el trabajo de Gastelbondo de acuerdo con los criterios de la ASGE (7) y la SBG (8); las dificultades en la estratificación del riesgo, obtenidas en el trabajo de Toro-Calle, a partir del modelo de la ASGE; la propuesta de una variable adicional predictora de riesgo de coledocolitiasis, basada en los casos y controles de Yurgaky, y la baja concordancia entre diferentes escalas para estimar el riesgo de morbimortalidad en pacientes con pancreatitis, solo son el reflejo de las complejidades de la predicción clínica, que son inherentes al encuentro con la biología (la cual es probabilística), a la información siempre incompleta, a los sesgos y a la validez interna de nuestros propios trabajos. Estas dificultades también son inherentes a la necesidad de perfeccionamiento continuo y de actualización de los modelos utilizados.

Desde Nostradamus -que era médico pero también astrólogo-, la predicción ha oscilado entre lo esotérico y la ciencia. Por tanto, para acercarnos con rigor científico a modelos de predicción clínica en un futuro próximo, se requiere con urgencia el desarrollo de estrategias apoyadas en el aprendizaje automático (machine learning).

Este enfoque permitirá incorporar variables y resultados predictores, o eliminar aquellas variables que no tengan un buen desempeño. Además, la enorme disponibilidad de datos que la red nos provee (big data) contribuye a tener mayores grados de certeza en la predictibilidad de los modelos, a estratificar mejor el riesgo, garantizar la rutas de atención requeridas y optimizar los resultados (8).

\section{REFERENCIAS}

1. McNicoll CF, Pastorino A, Farooq U, St Hill CR. Choledocholithiasis. In: StatPearls. Treasure Island (FL): StatPearls Publishing; June 18, 2020.

2. Williams E, Beckingham I, El Sayed G, Gurusamy K, Sturgess R, Webster G, Young T. Updated guideline on the management of common bile duct stones (CBDS). Gut. 2017;66(5):765-782. http://doi.org/10.1136/ gutjnl-2016-312317

3. Osío O, Zuleta JJ. Ronda clínica y epidemiológica. Iatreia. 2009;22(2):187-191.

4. Ebrahim M, Sorensen LT, Jorgensen LN, Kalaitzakis E. Current clinical algorithms for predicting common bile duct stones have only moderate accuracy. Dig Endosc. 2018;30(4):477-484. http://doi.org/10.1111/den.12994

5. Lee YH, Bang H, Kim DJ. How to Establish Clinical Prediction Models. Endocrinol Metab (Seoul).
2016;31(1):38-44. http://doi.org/10.3803/

EnM.2016.31.1.38

6. Collins GS, Reitsma JB, Altman DG, Moons KG. Transparent Reporting of a multivariable prediction model for Individual Prognosis Or Diagnosis (TRIPOD): The TRIPOD Statement. 2015;162(1):55-63. https://doi. org/10.7326/M14-0697

7. ASGE Standards of Practice Committee, Maple JT, BenMenachem T, Anderson MA, Appalaneni V, Banerjee S, Cash BD, Fisher L, Harrison ME, Fanelli RD, Fukami N, Ikenberry SO, Jain R, Khan K, Krinsky ML, Strohmeyer $\mathrm{L}$, Dominitz JA. The role of endoscopy in the evaluation of suspected choledocholithiasis. Gastrointest Endosc. 2010;71(1):1-9. http://doi.org/10.1016/j.gie.2009.09.041

8. Pencina MJ, Goldstein BA, D’Agostino RB. Prediction Models - Development, Evaluation, and Clinical 
Application. N Engl J Med. 2020;382(17):1583-1586. http://doi.org/10.1056/NEJMp2000589

9. Giljaca V, Gurusamy KS, Takwoingi Y, Higgie D, Poropat G, Štimac D, Davidson BR. Endoscopic ultrasound versus magnetic resonance cholangiopancreatography for common bile duct stones. Cochrane Database Syst Rev. 2015;2015(2):CD011549. http://doi. org/10.1002/14651858.CD011549 